\title{
Performance of arthroscopic irrigation systems assessed with automatic blood detection
}

\author{
G. J. M. Tuijthof $\cdot$ M. M. de Vaal • \\ I. N. Sierevelt • L. Blankevoort • M. P. J. van der List
}

Received: 29 April 2010/Accepted: 21 March 2011/Published online: 9 April 2011

(C) The Author(s) 2011. This article is published with open access at Springerlink.com

\begin{abstract}
Purpose During arthroscopies, bleeding episodes occur as a result of tissue damage. Irrigation systems assist in minimizing these disturbances. The performance of three arthroscopic irrigation systems in clearing bleeding episodes was evaluated objectively.

Methods One surgeon performed 99 shoulder arthroscopies using three irrigation systems: gravity infusion, single roller, and double roller pump. The three irrigation systems groups were matched postoperatively for type of surgery-acromioplasty, SLAP, release, rotator cuff repair and capsule repair, and operation duration. The recorded arthroscopies were analyzed for the presence of bleeding episodes with a special computer program that automatically detects the tinctures of red-colored blood. A least $20 \%$ of an arthroscopic image had to be covered with blood to qualify as bleeding episode.
\end{abstract}

G. J. M. Tuijthof $(\bowtie) \cdot$ M. M. de Vaal .

I. N. Sierevelt · L. Blankevoort

Orthopedic Research Center Amsterdam,

Department of Orthopedic Surgery, Academic Medical Center,

Meibergdreef 9, 1105 AZ Amsterdam, The Netherlands

e-mail: g.j.tuijthof@amc.uva.nl; g.j.m.tuijthof@tudelft.nl

G. J. M. Tuijthof

Department of BioMechanical Engineering,

fac. of 3ME, Delft University of Technology,

Mekelweg 2, 2628 CD Delft, The Netherlands

M. P. J. van der List

Bergman Medical Care, Prof. Bronkhorstlaan 10,

3723 MB Bilthoven, The Netherlands

M. P. J. van der List

Former Meander Medical Centre,

Baarn, The Netherlands
Results The median (min-max) presence of bleeding episodes as a percentage of the operation time was $6.6 \%$ $(0.0-43.6 \%)$ for gravity infusion, $3.7 \%(0.2-46.4 \%)$ for the single roller, and $3.3 \%(0.0-19.3 \%)$ for the double roller pump, respectively. The large variation could be attributed to the occurrence of arterial bleeding episodes during some procedures. No significant differences were found between the irrigation systems. For a subgroup including acromioplasties and releases, significant differences were found in favor of both roller pumps $(P<0.05)$.

Conclusions Overall, the roller pumps did not outperform gravity infusion. However, from the results, high-risk procedures for bleeding episodes were identified (acromioplasty and release of a frozen shoulder) that can benefit from the use of roller pumps. A clear view is essential to perform an arthroscopic procedure safely and efficiently. Level of evidence III.

Keywords Arthroscopy - Shoulder · Performance · Bleeding episode $\cdot$ View $\cdot$ Irrigation system

\section{Introduction}

Even if the arthroscope, light source, and camera are functioning properly, disturbances such as debris and bleeding episodes can block the arthroscopic view. Bleeding episodes occur due to damage of veins or arteries located in soft tissue or bone. Once bleeding episodes appear, surgeons need to regain a clear view quickly. Arthroscopic irrigation systems can assist in this. Different irrigation systems are on the market which can be categorized into three types $[15,17]$ : gravity infusion, single roller volumetric pumps, and double rollers volumetric pumps. In previous studies, its was indicated that irrigation 
systems have been evaluated by subjective measures only, such as visual clarity on a three-point scale or measures that were unrelated to the image quality, such as the number of fluid bags used for the operation [1, 4, 10, 11, $13,17]$. Objective evaluation of the performance of different types of arthroscopic irrigation systems is required as debate is continuing on the added value of the more advanced irrigation systems in relation to their purchase price. Therefore, we proposed to relate the irrigation performance of arthroscopic irrigation systems directly to the quality of the arthroscopic view as follows: The irrigation system performance is optimal if during an operation minimal visual disturbances are present [16, 17]. As bleeding episodes were identified as the most disturbing factor [14], the development of a special software program was initiated that can automatically detect the percentage of bleeding episodes frame by frame of a digitally recorded arthroscopic operation.

The goal was to compare the performance of three arthroscopic irrigation systems objectively in a routine clinical setting in clearing bleeding episodes by automatic blood detection with the proposed software program. Therefore, we posed the following hypothesis: The percentage of bleeding episodes in the arthroscopic view as detected by the software program is significantly smaller when using an arthroscopic roller pump compared to gravity infusion. In addition, two secondary hypotheses were formulated related to irrigation performance: (a) The time from the first presence of a bleeding episode till the disappearance of that bleeding episode is significantly smaller when using an arthroscopic roller pump compared to gravity infusion and (b) the use of instruments such as shaver, drill, and electrocautery device has a significant correlation with the presence of bleeding episodes.

\section{Materials and methods}

\section{Arthroscopic irrigation systems}

Three different types of irrigation systems were selected for performance evaluation (Fig. 1). The gravity infusion
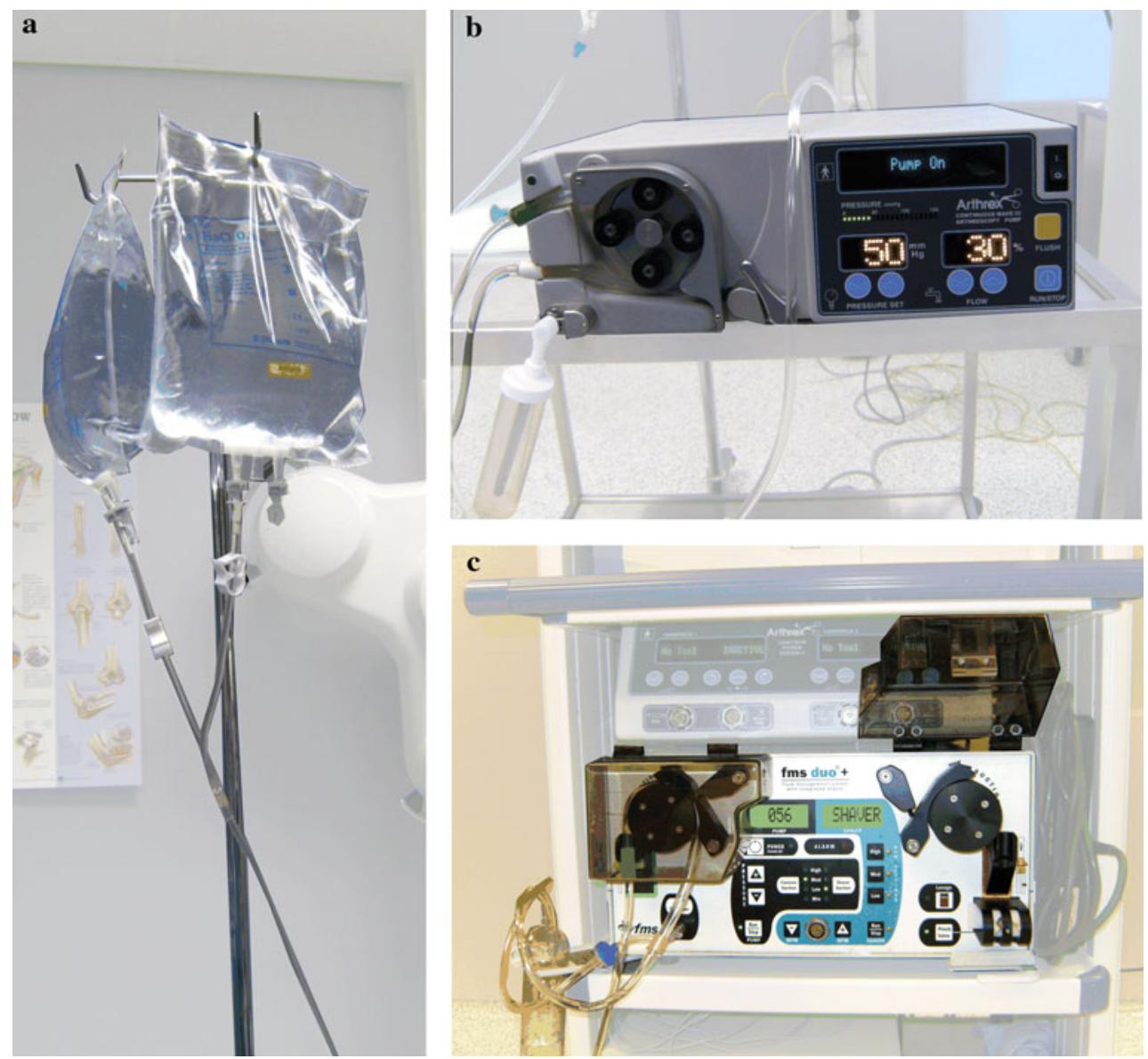

Fig. 1 Three arthroscopic irrigation systems were assessed for their performance with automated blood detection: a gravity infusion, b single roller pump, and c double roller pump. For the gravity infusion, two bags were routinely hung on a pole at a standard height, but only one was used at the same time 
creates a pressure by the height difference between the fluid bag and patient, which generates a fluid flow [8, 15]. The volumetric or roller pumps with a single roller work by peristaltic action causing a predetermined flow rate [12]. The volumetric pumps with double rollers can also create a constant flow, but as inflow and outflow are controlled by a separate roller pressure can be built up while maintaining a constant flow [15]. In this study, the single roller pump Continuous Wave III Arthroscopy pump (Arthrex Nederland B.V., St. Anthonis, The Netherlands) and the double roller pump FMS Duo pump (FMSGroup, Nice, France) were evaluated (Fig. 1). They both have the capability to control irrigation pressure. The settings of the roller pumps were chosen according to the guidelines of the supplier for performing a shoulder arthroscopy in a routine clinical setting. The following initial set pressures were used: $49 \mathrm{mmHg}$ for the gravity infusion, $50 \mathrm{mmHg}$ for the single roller pomp, and $56 \mathrm{mmHg}$ for the double roller pump. As part of the normal routine, $2 \mathrm{ml}$ adrenaline was injected in the first 3-1 fluid bag. The study deliberately did not interfere with the routine clinical protocols, to be able to generalize the results.

\section{Recording and matching of arthroscopies}

Within a period of 27 months (October 2006-January 2009), shoulder arthroscopies were recorded on digital video. The operations were recorded with a consumer digital video camera (JVC GR D93) on a notebook PC (HP Compaq NC6120 P-M $75060 \mathrm{~Gb} 512 \mathrm{Mb}$ ). The arthroscopic view was captured in AVI-format (image resolution: $720 \times 576$, frame rate: 25 images per second), which implied that all time durations could be determined with the accuracy of the frame rate $(0.04 \mathrm{~s})$ which was rounded to $0.1 \mathrm{~s}$. Each recording started when the surgeon inserted the arthroscope for the first time in the shoulder joint and ended when the arthroscope was pulled out to end the procedure, including recording of waiting time and portal exchanges. The patients were not notified of the recordings, because the operation was not influenced in any way by the recordings and the patients' identities could not be traced from the video.

For comparison of irrigation performance, all influencing factors should be kept constant or have equal influence among the groups. These factors were the surgeon, the condition of the arthroscope, light source and camera, the type of surgery, the operation time, and the duration of drill, shaver, electrocautery device, and scissors usage. Other instruments were found not to contribute to the presence or absence of bleeding, and their usage was not documented. One orthopedic surgeon (ML) performed all operations with arthroscopic instrument sets from one manufacturer (Arthrex Nederland B.V., St. Anthonis, The Netherlands). The condition of the camera, the light source, and the arthroscope was assumed constant, because one brand was used and the equipment was routinely inspected. The duration of the use of instruments was determined from the digitally recorded videos by timing the moment an instrument was in sight and until it disappeared and summing these episodes.

For type of surgery, we aimed at an equal number of removal procedures, i.e., acromioplasties and releases of frozen shoulders, and reconstruction procedures, i.e., rotator cuff and capsule repairs, in all three groups. For logistic reasons, initial randomization of the irrigation systems was performed per operation day, where one irrigation system was used during the entire day. As operation time and duration of the use of various instruments could only be determined postoperatively, matching was performed postoperatively. Based on the recorded operations and the matching criteria, the next period of recording was planned to complete triplets of matched shoulder operations. This process was repeated two additional times, until 33 triplets of shoulder arthroscopies were recorded (Table 1). In addition to the video recordings, the preoperative diastolic and systolic blood pressures of the patients were documented to determine whether one of these could be a predictor for increased bleeding episodes during surgery.

\section{Detection of bleeding episodes}

Previous studies found that the determination of the presence of bleeding episodes in the arthroscopic view showed a high observer agreement $(>0.83)$ [16], and that an arthroscopic image was not acceptable if it was covered by blood with an area of $25 \%$ or more [14]. With these results, a software program was designed (Matlab 7.5.0.342 (R2007b), (The Mathworks, Natick, Massachusettes, USA) that detects bleeding episodes automatically by filtering a combination of red, green and blue pixel levels representing the different red tinctures of blood. The digitally recorded operations were automatically analyzed frame by frame for the presence of red blood, without manual interference.

For this study, an arthroscopic image area of more than $20 \%$ covered with blood was used as a threshold level for the definition of the occurrence of a bleeding episode. Verification of the software routine was performed by visual comparison of a processed image with its original. Therefore, five hundred frames were randomly selected of each digitally recorded operation and analyzed for any significant deviations from the intended blood detection.

\section{Statistical analysis}

A power analysis was performed in a pilot study where five operations were matched in each group. The results gave a mean percentage of bleeding episode of the operation time 
Table 1 Ninety-nine shoulder arthroscopies were matched for type of surgery, instrument use, and operation time

\begin{tabular}{lllll}
\hline & & Gravity infusion & Single roller pump & Double roller pump \\
\hline SLAP (superior labrum anterior posterior) & Number of procedures [\#] & 2 & 2 & 2 \\
lesion repair & Mean (SD) OT [min.:sec.] & $29: 27(9: 17)$ & $37: 38(6: 31)$ & $25: 19(7: 53)$ \\
& Mean (SD) IU [\% of OT] & $5.5(5.0)$ & $2.0(0.0)$ & $5.5(6.4)$ \\
Acromioplasty and rotator cuff tear repair & Number of procedures [\#] & 1 & 1 & 1 \\
& Mean (SD) OT [min.:sec.] & $33: 16$ & $47: 53$ & $29: 20$ \\
& Mean (SD) IU [\% of OT] & 55.0 & 32.0 & 62.0 \\
Acromioplasty & Number of procedures [\#] & 14 & 14 & 14 \\
& Mean (SD) OT [min.:sec.] & $36: 52(13: 58)$ & $28: 51(7: 24)$ & $32: 12(7: 37)$ \\
& Mean (SD) IU [\% of OT] & $64.2(13.7)$ & $70.2(9.4)$ & $68.8(8.4)$ \\
Release frozen shoulder & Number of procedures [\#] & 4 & 4 & 4 \\
& Mean (SD) OT [min.:sec.] & $13: 29(4: 38)$ & $12: 28(4: 14)$ & $15: 28(8: 22)$ \\
& Mean (SD) IU [\% of OT] & $40.0(18.1)$ & $45.8(19.5)$ & $50.5(12.4)$ \\
Rotator cuff tear repair & Number of procedures [\#] & 5 & 5 & 5 \\
& Mean (SD) OT [min.:sec.] & $42: 06(17: 44)$ & $49: 24(23: 44)$ & $37: 44(20: 27)$ \\
& Mean (SD) IU [\% of OT] & $6.6(11.0)$ & $14.8(13.6)$ & $14.4(18.1)$ \\
& Number of procedures [\#] & 7 & 7 & 7
\end{tabular}

OT Operation time, $I U$ instrument usage

of $18 \%$ (SD 14\%) for the gravity infusion, $12 \%$ (SD 17\%) for the single roller pump, and 5\% (SD 4\%) for the double roller pump. A difference between the irrigation system groups of $10 \%$ was considered clinical relevant. This required 33 operations per irrigation system group as calculated with the median standard deviation of $14 \%$ and a power of $80 \%(\alpha=0.05)$.

The primary outcome measure was the amount of bleeding episodes expressed as percentage of operation time (OT). The absolute total bleeding episode time per operation in seconds (ATB) was presented as well for convenience of interpretation (Table 2). The second outcome measure was the mean clinically relevant bleeding episode time (MCRB), which was defined as the mean bleeding episode time of all bleeding episodes in an operation which were present for $2 \mathrm{~s}$ or longer. The results of an expert opinion panel demonstrated that the majority would undertake action to control a bleeding episode after a waiting period of $2 \mathrm{~s}$ or longer [14].

Statistical analysis was performed with SPSS 15.0 (SPSS Inc., Chicago, Illinois, USA). The presence of a normal distribution was determined with the KolmogorovSmirnov tests. Nonparametric Kruskal-Wallis (KW) tests were performed to identify significant differences for all matched operations $(P<0.05)$. Post hoc Mann-Whitney $U$ (MWU) tests were used to highlight the significant internal differences between the three irrigation system groups and between removal and reconstruction procedures. As all data are expressed as medians, the median percentage of operation time does not correspond with the absolute times as indicated by ATB or MCRB. Spearman's correlation coefficients were determined between ATB and the duration of shaver, drill, electrocautery device, and scissors expressed as percentage of the operation time and between the outcome measures and the preoperative diastolic and systolic blood pressures. Finally, the presence of significant differences was assessed between the type of procedure and the duration of the various instruments.

\section{Results}

An example of one of the automated red-color detection of one operation (arthroscopic shoulder release) is shown (Fig. 2). For this particular operation, bleeding episodes apparently occur at the start and at the end of the operation.

The main result is that the median percentage of bleeding episodes for all matched operations is not significantly different between the three irrigation groups (Table 2 first row). A large variation in ATB is present for the gravity infusion (up to $15 \mathrm{~min}$. and $28 \mathrm{~s}$.) compared to the roller pumps (Table 2).

The presence of bleeding episodes is significantly higher for removal procedures (median of 7.8\%) compared to reconstruction procedures (median of $0.5 \%$ ) (MWU, $P<0.01$ ) (Fig. 3). The removal procedures show significant differences between the irrigation systems (KW, $P<0.05$ ) in favor of the roller pumps for ATB (Table 2 
Table 2 Results of the four outcome measures for all operations together and for the two subgroups removal and reconstruction

\begin{tabular}{llccc}
\hline & & Gravity infusion & Single roller pump & $\begin{array}{c}\text { Double roller } \\
\text { pump }\end{array}$ \\
\hline All operations & Median (min.-max.) bleeding episode [\% of & $6.6(0.0-43.6)$ & $3.7(0.2-46.4)$ & $3.3(0.0-19.3)$ \\
& OT] & & & \\
& Median (min.-max.) ATB [sec.] & $75.0(0.0-927.7)$ & $65.3(5.7-457.1)$ & $54.8(0.0-339.6)$ \\
& Median (min.-max.) of MCRB [sec.] & $4.8(0.0-20.3)$ & $4.3(0.0-9.8)$ & $3.7(0.0-7.5)$ \\
Subgroup removal procedures & Median (min.-max.) ATB [sec.] & $230.0(34.3-927.7)^{*}$ & $116.1(31.2-457.1)^{*}$ & $88.2(42.0-281.5)^{*}$ \\
& Median (min.-max.) of MCRB [sec.] & $5.4(2.9-7.2)$ & $4.2(2.9-8.2)$ & $4.3(3.2-7.5)$ \\
Subgroup reconstruction & Median (min.-max.) ATB [sec.] & $6.5(0.0-268.9)$ & $12.3(5.7-324.5)$ & $4.6(0.0-137.2)$ \\
procedures & Median (min.-max.) of MCRB [sec.] & $0.0(0.0-7.4)$ & $4.0(0.0-6.6)^{* *}$ & $0.0(0.0-4.2)^{* *}$ \\
\hline
\end{tabular}

If no symbols are added to the values, no significant differences were present. For '*', a significant difference $($ MWU, $P<0.05)$ was present between the gravity infusion and both types of roller pumps, whereas no significant difference was present between the roller pumps. For '**', a significant difference (MWU, $P<0.05$ ) was present between the single roller and the double roller pump solely. OT Operation time, ATB absolute total bleeding episode time per operation, $M C R B$ mean clinically relevant bleeding episode time per operation

Fig. 2 Result of automated detection of bleeding episodes of one arthroscopic frozen shoulder release operation. The percentage of red-colored arthroscopic image area per video frame is automatically detected and plotted against time (gray line). The threshold level of $20 \%$ is indicated by the striped horizontal line. All frames that demonstrate more than $20 \%$ red image area coverage are included to determine the presence of bleeding episodes. One bleeding episode is indicated by the vertical black lines

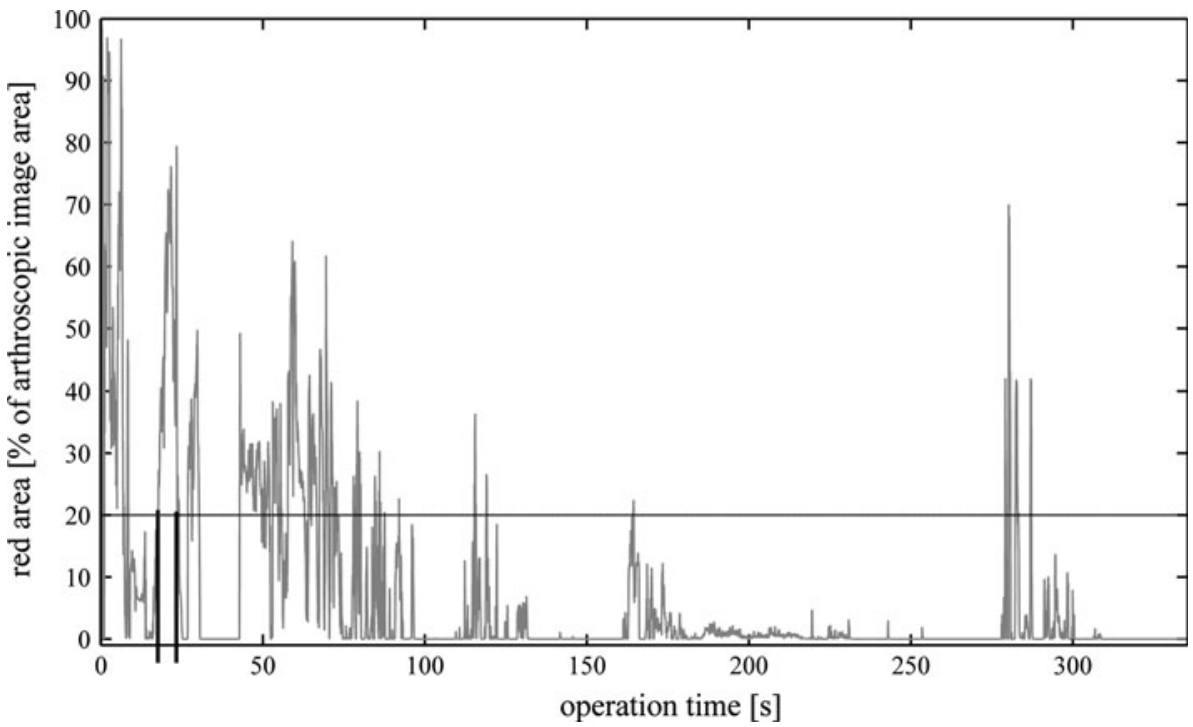

fourth row) and in favor of the double roller pump versus the gravity infusion for overall percentage of bleeding episodes (MWU, $P<0.05$ ) (Fig. 3). The MCRB of the reconstruction procedures is significantly higher for the single roller pump compared to the double roller pump (MWU, $P<0.05$ ) (Table 2 last row).

Spearman correlation coefficients between ATB and the duration of shaver usage, drill usage, and electrocautery device are significant $(P<0.01): r=0.52, r=0.46$, $r=0.66$, respectively. A significant correlation was neither present between the duration of scissors usage and ATB nor between the preoperative diastolic or systolic blood pressures and any of the outcome measures.

The total instrument use is significantly higher for the removal procedures compared to reconstruction and other procedures (KW, $P<0.05$ ). The percentage of shaver usage is significantly different for all three procedure groups with the highest for removal procedures and lowest value for reconstruction procedures (MWU, $P<0.05$ ). Drill use and electrocautery device use are significantly higher in removal procedures compared to both reconstruction and other procedures (MWU, $P<0.05$ ).

\section{Discussion}

The most important finding of the present study was that the median percentage of bleeding episodes for all matched operations is not significantly different between the three irrigation groups, which implies that the primary hypothesis (roller pumps show lower percentage of bleeding episodes than the gravity infusion) is rejected.

In this study, an objective evaluation of irrigation system performance was performed based on automated detection of bleeding episodes in the arthroscopic view. This way, irrigation system performance was directly 


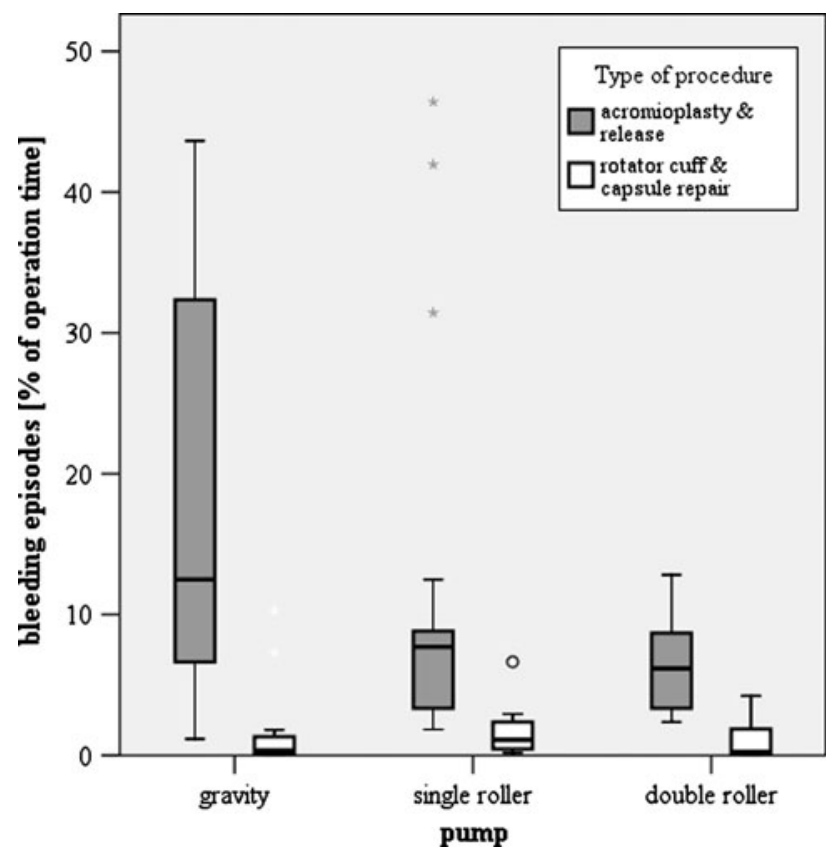

Fig. 3 Boxplot is shown of the bleeding episode time as percentage of the operation time. The results are shown for the subgroups removal procedures consisting of 18 matched operations and reconstruction procedures consisting of 12 matched operations (Tables 1, 2)

coupled to the arthroscopic view. The roller pumps did not outperform the gravity infusion. One explanation could be the considerable variation in the presence of bleeding episodes in a similar type operation, which ranged from $0 \%$ to a maximum of $46 \%$ (Table 2). Particularly arterial bleeding episodes could be the major cause. If they occur, the irrigation systems cannot control these bleeding episodes completely. Notice that the use of the shaver and the drill causes bleeding episodes in contrary to the use of the electrocautery device, which is used to control arterial bleeding episodes.

Two subgroups were analyzed separately. As expected, more bleeding episodes occur for the removal procedures. In these procedures, treatment exists of removal of bone or soft tissue, which causes both venous and arterial bleeding episodes (Table 2; Fig. 3). When performing a reconstruction procedure, the presence of bleeding episodes is small as a majority of the time is spent on stitching tissue (Table 2; Fig. 3). Concluding the primary hypothesis was accepted for removal procedures and rejected for reconstruction procedures (Fig. 3).

Overall, the MCRB is not significantly different between roller pumps and the gravity infusion, which implies that the roller pumps do not outperform the gravity infusion in minimizing the time from the first presence of a bleeding episode till the disappearance of that bleeding episode. The median MCRB for all three irrigation system groups is larger than the $2 \mathrm{~s}$ that surgeons are willing to wait [14], which implies that there is room for improvement of the irrigation systems.

No correlation is present between the occurrence and severity of the bleeding episodes and the patients' preoperative blood pressure. Thus, preoperative blood pressure is of no value when trying to estimate an increased risk of bleeding episodes, preoperatively. However, a strong correlation is found between instrument use and presence of bleeding episodes on one hand and a significant difference in instrument use for different types of procedure on the other hand. Combining those results, high-risk procedures for bleeding episodes can be identified: acromioplasty and release of a frozen shoulder. For these types of procedures, it is recommended to use a roller pump. The reason is that these irrigations systems are better capable of keeping the intra-articular pressure at a higher and constant level than the gravity infusion, which is important to assist the control of (arterial) bleeding episodes.

This study has its limitations. Due to the considerable period in which the operations were recorded, the surgeon might have tuned his operative techniques in time. However, as the operations were recorded for all three irrigation systems in each period, the expected bias in this study is small. As none of the irrigation systems perform fully automatic, surgeons act as system controller, and their capabilities influence pump performance as well. This aspect was not studied but could be performed in a simulated setting (e.g., [18]) using objective performance measures [2, 5] or global rating scales [6, 7]. Additionally, arthroscopic irrigation systems can also be used to assist in other treatments such as joint lavage [3,9], which might require other performance capacities. As final matching had to take place postoperatively, the possibility of randomization was limited. Investigator bias was minimized by using strict matching criteria. The surrounding tissue in the joint was sometimes inflamed. This tissue shows red colored patched in the same red tincture palette as for the colors of blood but should not be marked as a blood. By choosing a relatively high threshold level of $20 \%$ of the arthroscopic image is to qualify as bleeding, the influence of this phenomenon was assumed to be low. Additionally, as the operations were matched, the eventual presence of inflammation would be evenly distributed among the three irrigation system groups and therefore have little influence on the study results. The duration of operation time and instrument usage were not automatically determined, but by visual inspection of the video recordings. No test-retest reliability measurements were performed, but the observer had the opportunity to stop and replay the videos which enhanced accurate determination of instrument presence. Finally, no registration of pressure or flow during the operations was performed. Therefore, no detailed analysis 
of the controlling actions or pressure levels at bleeding episodes could be performed. To the best of our knowledge, no comparable studies have been performed in this field, which limits adequate positioning within the existing literature. Clear view is important to perform an arthroscopic operation safely and efficiently. With the results of this study, surgeons are encouraged to use roller pumps when performing arthroscopic acromioplasty and release of a frozen shoulder to minimize the risk of inadequate arthroscopic view during the operation. This recommendation is supported by the findings in other studies where the operation time was lower when using more advanced pump systems in technically more demanding procedures $[1,13]$.

\section{Conclusion}

Concluding, arthroscopic system irrigation performance was objectively assessed in relation to the arthroscopic view. The roller pumps did not outperform the gravity infusion. However, when the results are combed, high-risk procedures for bleeding episodes could be identified (acromioplasty and release of a frozen shoulder) that can benefit from the use of roller pumps.

Acknowledgments This work was supported by the Technology Foundation STW, Applied Science Division of NWO, and the Technology Program of the Ministry of Economic Affairs, The Netherlands. No financial support was received from any company. The authors wish to thank D. Nagel and P. Vatankhah for recording part of arthroscopic operations, and all personnel of the day care operating theaters in Bergman Medical Care, Bilthoven, The Netherlands and Meander Medical Centre, Baarn, The Netherlands for their cooperation and patience.

Conflict of interest Supported by outside funding or grant(s) from Technology Foundation STW, applied science division of NWO, and the technology program of the Ministry of Economic Affairs, The Netherlands. None of the researchers or an affiliated institute has received (or agreed to receive) from a commercial entity something of value related in any way to this manuscript or research. The authors did not have any financial and personal relationships with other people or organizations that could inappropriately influence (bias) their work. None of the supporting organizations was involved in the study design, the collection, analysis and interpretation of data, in the writing of the manuscript and in the decision to submit the manuscript for publication.

Open Access This article is distributed under the terms of the Creative Commons Attribution Noncommercial License which permits any noncommercial use, distribution, and reproduction in any medium, provided the original author(s) and source are credited.
2. Chami G, Ward JW, Phillips R, Sherman KP (2008) Haptic feedback can provide an objective assessment of arthroscopic skills. Clin Orthop Relat Res 466:963-968

3. Di Gennaro GL, Bettuzzi C, Antonioli D, Lampasi M, Rotini R, Donzelli O (2008) Arthroscopic treatment of septic arthritis of the shoulder in a 6-year-old boy. Orthopedics 31:809

4. Dolk T, Augustini BG (1989) Three irrigation systems for motorized arthroscopic surgery: a comparative experimental and clinical study. Arthroscopy 5:307-314

5. Howells NR, Brinsden MD, Gill RS, Carr AJ, Rees JL (2008) Motion analysis: a validated method for showing skill levels in arthroscopy. Arthroscopy 24:335-342

6. Howells NR, Gill HS, Carr AJ, Price AJ, Rees JL (2008) Transferring simulated arthroscopic skills to the operating theatre: a randomised blinded study. J Bone Joint Surg $\mathrm{Br}$ 90:494-499

7. Insel A, Carofino B, Leger R, Arciero R, Mazzocca AD (2009) The development of an objective model to assess arthroscopic performance. J Bone Joint Surg Am 91:2287-2295

8. Morgan CD (1987) Fluid delivery systems for arthroscopy. Arthroscopy 3:288-291

9. Moseley JB, O’Malley K, Petersen NJ, Menke TJ, Brody BA, Kuykendall DH, Hollingsworth JC, Ashton CM, Wray NP (2002) A controlled trial of arthroscopic surgery for osteoarthritis of the knee. N Engl J Med 347:81-88

10. Muellner T, Menth-Chiari WA, Reihsner R, Eberhardsteiner J, Engebretsen L (2001) Accuracy of pressure and flow capacities of four arthroscopic fluid management systems. Arthroscopy 17:760-764

11. Ogilvie-Harris DJ, Weisleder L (1995) Fluid pump systems for arthroscopy: a comparison of pressure control versus pressure and flow control. Arthroscopy 11:591-595

12. Ozcelik S (2006) Drug infusion systems. In: Webster J (ed) Encyclopedia of medical devices and instrumentation. Wiley, New York, pp 495-508

13. Sieg R, Bear R, Machen MS, Owens BD (2009) Comparison of operative times between pressure and flow-control pump versus pressure-control pump for ACL reconstruction. Orthopedics $32: 727$

14. Tuijthof GJ, Abbink M, Sierevelt IN, van Dijk CN (2009) Multirater agreement on arthroscopic image quality. Proc Inst Mech Eng [H] 223:179-187

15. Tuijthof GJ, Dusee L, Herder JL, van Dijk CN, Pistecky PV (2005) Behavior of arthroscopic irrigation systems. Knee Surg Sports Traumatol Arthrosc 13:238-246

16. Tuijthof GJ, Sierevelt IN, van Dijk CN (2007) Disturbances in the arthroscopic view defined with video analysis. Knee Surg Sports Traumatol Arthrosc 15:1101-1106

17. Tuijthof GJ, van den Boomen H, van Heerwaarden RJ, van Dijk CN (2008) Comparison of two arthroscopic pump systems based on image quality. Knee Surg Sports Traumatol Arthrosc 16:590-594

18. Tuijthof GJ, van Sterkenburg MN, Sierevelt IN, van Oldenrijk J, van Dijk CN, Kerkhoffs GM (2010) First validation of the PASSPORT training environment for arthroscopic skills. Knee Surg Sports Traumatol Arthrosc 18:218-224

\section{References}

1. Ampat G, Bruguera J, Copeland SA (1997) Aquaflo pump vs. FMS 4 pump for shoulder arthroscopic surgery. Ann R Coll Surg Engl 79:341-344 